Canad. Math. Bull. Vol. 21 (1) 1978

\title{
HOMOMORPHISMS BETWEEN LATTICES OF ZERO-SETS
}

\author{
BY \\ S. BROVERMAN ${ }^{1}$
}

\begin{abstract}
For a completely regular Hausdorff topological space $X$, let $Z(X)$ denote the lattice of zero-sets of $X$. If $\tau$ is a continuous map from $X$ to $Y$, then there is a lattice homomorphism $\tau^{\prime}$ from $Z(Y)$ to $Z(X)$ induced by $\tau$ which is defined by $\tau^{\prime}(A)=\tau^{\leftarrow}(A)$. A characterization is given of those lattice homomorphisms from $Z(Y)$ to $Z(X)$ which are induced in the above way by continuous function from $X$ to $Y$.
\end{abstract}

1. Introduction. The theory of duality linking topology and algebra has been studied in depth in the past. Most notably in this area, is the work of M. H. Stone in [3], which describes the duality between compact, Hausdorff, 0dimensional spaces (i.e. spaces with a base of open-and-closed sets) and Boolean algebras. In particular, it is shown that if $X$ and $Y$ are compact, Hausdorff, 0-dimensional spaces and $B(X)$ and $B(Y)$ are their Boolean algebras of clopen sets then if $t: B(Y) \rightarrow B(X)$ is a homomorphism such that $t(Y)=X$, then there is a continuous map $f: X \rightarrow Y$ such that $t(A)=f^{\leftarrow}(A)$ for all $A \in B(Y)$. In chapter 10 of [1] this aspect of duality is studied where the algebraic object is $C(X)$, the ring of continuous functions of a completely regular, Hausdorff space. It is shown that if $X$ and $Y$ are realcompact spaces and if $t: C(Y) \rightarrow C(X)$ is a homomorphism such that $t\left(\left(1_{Y}\right)=1_{X}\right.$ (where 1 denotes the constant function whose range is $\{1\})$, then there is a continuous function $f: X \rightarrow Y$ such that $t(g)=g \circ f$ for all $g \in C(Y)$.

In this paper we consider the lattice $Z(X)$, of zero-sets of a completely regular, Hausdorff space and characterize these lattice homomorphisms between zero-set lattices that arise, in the natural way described above, from continuous functions.

1.1. Definition. (a) Let $X$ and $Y$ be spaces and let $Z(X)$ and $Z(Y)$ denote their respective zero-set lattices. By a $\sigma$-homomorphism $t$ from $Z(Y)$ to $Z(X)$

AMS Subj. Class: Primary 54C05, 54C50.

Secondary 54D35, 54D60

Key words and phrases: zero-set lattice, lattice homomorphism, $z$-ultrafilter, realcompact, Stone-Cech compactification.

Received by the editors November 30, 1976 and in revised form, June 30, 1977.

${ }^{1}$ This research was partially supported by a scholarship from the National Research Council of Canada. 
we will mean a lattice homomorphism $t: Z(Y) \rightarrow Z(X)$ such that $t$ preserves countable meets (i.e. if $\left\{A_{i}\right\}_{i=1}^{\infty} \subseteq Z(Y)$, then $\left.t\left(\bigcap_{i=1}^{\infty} A_{i}\right)=\bigcap_{i=1}^{\infty} t\left(A_{i}\right)\right)$, and such that $t(\phi)=\phi, t(Y)=X$.

(b) Let $X$ and $Y$ be spaces and let $\tau: X \rightarrow Y$ be a continuous map. Then the "homomorphism induced by $\tau$ " is the map $\tau^{\prime}: Z(Y) \rightarrow Z(X)$ defined by $\tau^{\prime}(A)=$ $\tau^{\leftarrow}(A)$ for $A \in Z(Y)$.

Note that in 1.1(b) $\tau^{\prime}$ is actually a $\sigma$-homomorphism in the sense of $1.1(\mathrm{a})$. In section 2 it is shown that these are precisely the homomorphisms induced by continuous maps.

The notation will be that of [1]. The set of integers is denoted by $N$. All spaces discussed are assumed to be completely regular and Hausdorff.

2. The homomorphism induced by a continuous map. Before getting to the main characterization theorem, some results of general interest on the induced homomorphism are obtained.

2.1 Definition. (a) A subspace $W$ of $X$ is $G_{\delta}$-dense in $X$ if every $G_{\delta}$ in $X$ meets $W$ in a non-empty set.

(b) A subspace $W$ of $X$ is $z$-embedded in $X$ if every zero-set in $W$ is the restriction to $W$ of a zero-set in $X$.

2.2. Theorem. Let $\tau$ be a continuous map from $X$ to $Y$ and let $\tau^{\prime}$ be the induced homomorphism from $Z(Y)$ to $Z(X)$. Then

(a) $\tau^{\prime}$ is one-to-one iff $\tau(X)$ is $G_{\delta}$-dense in $Y$, and

(b) $\tau^{\prime}$ is onto iff $\tau$ is a homeomorphism onto a $z$-embedded subset of $Y$.

Proof. (a) Necessity. Suppose $\tau(X)$ is not $G_{\delta}$-dense in $Y$. Then there is a non-empty $G_{\delta}$ set $H=\bigcap_{i \in N} U_{i}$ in $Y$ (where $U_{i}$ is open in $Y$ for all $i \in N$ ) such that $H \cap \tau(X)=\phi$. Let $y \in H$. Then there is a zero-set $Z$ in $Y$ such that $y \in Z \subseteq H$ (for each $i \in N$ there is a zero-set $Z_{i}$ in $Y$ such that $y \in Z_{i} \subseteq U_{i}$ since $Y$ is completely regular, thus $\left.y \in Z=\bigcap_{i \in N} \subseteq Z_{i} \bigcap_{i \in N} U_{i}=H\right)$. Hence $\tau^{\prime}(Z)=$ $\tau^{\leftarrow}(Z)=\phi=\tau^{\leftarrow}(\phi)=\tau^{\prime}(\phi)$ and thus $\tau^{\prime}$ is not one-to-one.

Sufficiency. Suppose $\tau^{\prime}$ is not one-to-one. Then there exist $Z_{1}, Z_{2} \in Z(Y)$ such that $\tau^{\leftarrow}\left(Z_{1}\right)=\tau^{\leftarrow}\left(Z_{2}\right)$ but $Z_{1} \neq Z_{2}$. Let $p \in Z_{1}-Z_{2}$. Then there is a $Z_{3} \in$ $Z(Y)$ such that $p \in Z_{3}$ and $Z_{3} \cap Z_{2}=\phi$ (as $Y-Z_{2}$ is a neighborhood of $p$ ). Let $Z=Z_{1} \cap Z_{3}$. Then $\tau^{\prime}(Z)=\tau^{\leftarrow}\left(Z_{1} \cap Z_{3}\right)=\tau^{\prime}\left(Z_{1}\right) \cap \tau^{\leftarrow}\left(Z_{3}\right)=\tau^{\leftarrow}\left(Z_{2}\right) \cap \tau^{\leftarrow}\left(Z_{3}\right)=$ $\tau^{\leftarrow}\left(Z_{2} \cap Z_{3}\right)=\tau^{\leftarrow}(\phi)=\phi$. Thus $Z \cap \tau(X)=\phi$ and hence $\tau(X)$ is not $G_{\delta}$ dense in $Y$.

(b) This result has been obtained independently by Mandelker in [2, p. 619], to which the reader is referred for a proof.

2.3. Definition. Let $X$ be a space. A $z$-filter $\mathscr{F}$ on $X$ is a filter on the lattice $Z(X)$. That is $\mathscr{F} \subseteq Z(X)$ such that i) $\phi \notin \mathscr{F}$, ii) if $Z_{1}, Z_{2} \in \mathscr{F}$ then $Z_{1} \cap Z_{2} \in \mathscr{F}$, and iii) if $Z_{1} \in \mathscr{F}$ and $Z \in Z(X)$ such that $Z_{1} \subseteq Z$, then $Z \in \mathscr{F}$. A $z$-filter $\mathscr{F}$ on $X$ 
is prime if $Z_{1} \cup Z_{2} \in \mathscr{F}$ implies that either $Z_{1} \in \mathscr{F}$ or $Z_{2} \in \mathscr{F}$. A $z$-ultrafilter is a maximal $z$-filter. A real $z$-ultrafilter is a $z$-ultrafilter closed under countable intersection (i.e. if $\left\{Z_{i}\right\}_{i \in N} \subseteq \mathscr{F}$, then $\bigcap_{i \in N} Z_{i} \in \mathscr{F}$ ).

2.4. Remark. If $\{p\}$ is the one point space, then $Z(\{p\})=\{\phi,\{p\}\}$ is the two-point lattice which will be referred to here as $\{0,1\}$. There is a one-to-one correspondence between $\sigma$-homomorphisms from $Z(Y)$ onto $\{0,1\}$ and real $z$-ultrafilters on $Y$. Clearly if $\mathscr{F}$ is a real $z$-ultrafilter on $Y$, then $t: Z(Y) \rightarrow$ $\{0,1\}$ defined by $t(Z)=1$ if $Z \in \mathscr{F}, t(Z)=0$ otherwise, is a $\sigma$-homomorphism. On the other hand, if $t: Z(Y) \rightarrow\{0,1\}$ is a $\sigma$-homomorphism, then clearly $\mathscr{F}=t^{\leftarrow}(\{1\})$ is a prime $z$-filter on $Y$. Since $t$ is a $\sigma$-homomorphism, $\mathscr{F}$ is closed under countable intersection. Thus, by $[1,7 \mathrm{H} 4], \mathscr{F}_{F}$ is a real $z$-ultrafilter. It is also apparent that there is a one-to-one correspondence between all homomorphisms from $Z(Y)$ to $\{0,1\}$ and all prime $z$-filters on $Y$.

2.5. THEOREM. Let $Y$ be a realcompact space and let $t$ be a homomorphism from $Z(Y)$ to $Z(X)$. The following are equivalent.

(i) $t$ is a $\sigma$-homomorphism.

(ii) $t=\tau^{\prime}$ for a unique continuous map $\tau: X \rightarrow Y$.

Proof. (ii) implies (i). We have already noted in the introduction that this implication is true.

(i) implies (ii). Let $x \in X$. Let $A_{x}=\{Z \in Z(X) \mid x \in Z\}$. Then $A_{x}$ is a real $z$-ultrafilter on $X$. Let $t^{\leftarrow}\left(A_{x}\right)=\left\{Z \in Z(Y) \mid t(Z) \in A_{x}\right\}$. Then $t^{\leftarrow}\left(A_{x}\right)$ is clearly a prime $z$-filter on $Y$. Furthermore, $t^{\leftarrow}\left(A_{x}\right)$ is closed under countable intersection (since $t$ is a $\sigma$-homomorphism). Thus, by [1,7H4], $t^{\leftarrow}\left(A_{x}\right)$ is a real $z$-ultrafilter on $Y$. Since $Y$ is realcompact, there is a unique $y \in Y$ such that $\cap \mathrm{t}^{\leftarrow}\left(\mathrm{A}_{\mathrm{x}}\right)=\{\mathrm{y}\}$. Define $\tau(x)=y$. Then $\tau$ is a well-defined map from $X$ to $Y$.

Let $Z \in Z(Y)$. Then $t(Z)=\tau^{\leftarrow}(Z)$, for $x \in t(Z)$ iff $t(Z) \in A_{x}$ iff $Z \in t^{\leftarrow}\left(A_{x}\right)$ iff $x \in \tau^{\leftarrow}(Z)$. This shows that $t=\tau^{\prime}$ and $\tau$ is continuous, as every closed subset of $Y$ is an intersection of zero-sets.

If $\sigma: X \rightarrow Y$ such that $\sigma^{\prime}=\tau^{\prime}=t$, then $\sigma^{\rightarrow}(Z)=\tau^{\leftarrow}(Z)$ for every $Z \in Z(Y)$. Thus $\sigma^{\leftarrow}(\{y\})=\sigma^{\leftarrow}\left(\bigcap A_{y}\right)=\tau^{\leftarrow}\left(\bigcap A_{y}\right)=\tau^{\leftarrow}(\{y\})$. Hence $\sigma=\tau$, and $\tau$ is unique.

The condition " $Y$ is realcompact" in 2.5 cannot be dropped because, as is shown in $[1,8 \mathrm{D} 2], Z(X) \cong Z(v X)$ (lattice isomorphic by the isomorphism $Z \rightarrow c l_{v X} Z$, where $v X$ denotes the Hewitt realcompactification of $X$ ) for any space $X$, thus $Z(X)$ does not distinguish between $X$ and $v X$. This immediately gives the following corollary to 2.5 .

2.6. Corollary. If $t$ is a $\sigma$-homomorphism from $Z(Y)$ to $Z(X)$, then there is a unique continuous map $\tau: X \rightarrow v Y$ such that $t(Z)=\tau^{\leftarrow}\left(\mathrm{cl}_{\mathrm{v}} Z\right)$ for all $Z \in$ $Z(Y)$. 
Theorem 2.5 also yields the following results.

2.7. Corollary. If $X$ and $Y$ are two spaces then $Z(X) \cong Z(Y)$ (lattice isomorphic) iff $v X \cong v Y$.

Proof. Sufficiency. This follows from the fact, noted above, that $Z(X) \cong$ $Z(v X)$ and $Z(Y) \cong Z(v Y)$.

Necessity. Let $t: Z(v Y) \rightarrow Z(v X)$ be an isomorphism. Then $s: Z(v X) \rightarrow$ $Z(v Y)$ defined by $s(Z)=t^{\leftarrow}(Z)$ is also an isomorphism. By 2.5 there are continuous maps $\tau: v X \rightarrow v Y$ and $\sigma: v Y \rightarrow v X$ such that $t=\tau^{\prime}$ and $s=\sigma^{\prime}$ (as an isomorphism is, in particular, a $\sigma$-homomorphism). Then $\sigma^{\prime} \circ \tau^{\prime}=s \circ t=$ $1_{Z(v Y)}$ (the identity map) and $\tau^{\prime} \circ \sigma^{\prime}=t \circ s=1_{Z(v X)}$, i.e. $\sigma^{\prime} \circ \tau^{\prime}=\left(1_{v Y}\right)^{\prime}$ and $\tau^{\prime} \circ \sigma^{\prime}=\left(1_{v X}\right)^{\prime} \quad$ But $\quad \sigma^{\prime} \circ \tau^{\prime}=(\tau \circ \sigma)^{\prime} \quad\left(\right.$ as $\quad \sigma^{\prime} \circ \tau^{\prime}(Z)=\sigma^{\leftarrow}\left(\tau^{\leftarrow}(Z)\right)=(\tau \circ \sigma)^{\leftarrow}(Z)=$ $\left.(\tau \circ \sigma)^{\prime}(Z)\right)$, and $\tau^{\prime} \circ \sigma^{\prime}=(\sigma \circ \tau)^{\prime}$. Thus $(\tau \circ \sigma)^{\prime}=\left(1_{v Y}\right)^{\prime}$ and $(\sigma \circ \tau)^{\prime}=\left(1_{v X}\right)^{\prime}$. By uniqueness $\tau^{\circ} \sigma=1_{v Y}, \sigma \circ \tau=1_{v X}$. Thus $\sigma$ and $\tau$ are homeomorphisms.

2.8. Corollary. vY contains a continuous image of $X$ iff $Z(X)$ contains a $\sigma$-homomorphic image of $Z(Y)$.

2.2 together with 2.5 yield the following results.

2.9. Corollary. $Z(X)$ contains a $\sigma$-isomorphic copy of $Z(Y)$ iff $v Y$ contains a $G_{\delta}$-dense continuous image of $X$.

2.10. Corollary. $v Y$ contains a $Z$-embedded copy of $X$ iff $Z(X)$ is a $\sigma$-homomorphic image of $Z(Y)$.

Results 2.5 to 2.10 are the $Z(X)$-analogues of 10.6 and 10.9 in [1].

3. The continuous map induced by a homomorphism. In section 2 we were concerned only with $\sigma$-homomorphisms. We now show that any lattice homomorphism $t: Z(Y) \rightarrow Z(X)$ (such that $t(Y)=X$ and $t(\phi)=\phi$ ) induces a continuous map $\tau: \beta X \rightarrow \beta Y$.

3.1. Definition. Let $X$ be a space. Let

$$
\begin{aligned}
M^{p} & =\left\{Z \in Z(X) \mid p \in c l_{\beta X} Z\right\} \text { and } \\
0^{p} & =\left\{Z \in Z(X) \mid c l_{\beta X^{2}} \text { is a neighborhood in } \beta X \text { of } p\right\} .
\end{aligned}
$$

3.2. Proposition. Let $t: Z(Y) \rightarrow Z(X)$ be a lattice homomorphism. Then there exists a continuous map $\tau: \beta X \rightarrow \beta Y$ such that $\tau^{\leftarrow}\left(c_{\beta Y} Z\right) \supseteq c l_{\beta X} t(Z)$. For a given $Z \in Z(Y)$, the above containment is equality if $t^{\leftarrow}\left(M^{p}\right)=M^{\tau(p)}$ for every $p \in \tau\left(c l_{\beta Y} Z\right)$ (i.e. is $t^{\leftarrow}\left(M^{p}\right)$ is an ultrafilter for every $p \in \tau^{\leftarrow}\left(c_{\beta Y} Z\right)$ ).

Proof. Let $t: Z(Y) \rightarrow Z(X)$ be as hypothesized and let $x \in \beta X$. Then $t^{\leftarrow}\left(M^{x}\right)$ is a prime $z$-filter on $Y$. Thus, by [1.2.11], $t^{\leftarrow}\left(M^{x}\right)$ is contained in a unique $z$-ultrafilter $M^{p}$, on $Y$. Let $\tau: \beta X \rightarrow \beta Y$ be defined by $\tau(x)=p$. Let $Z \in Z(Y)$. Let $x \in c l_{\beta X} t(Z)$. Then $t(Z) \in M^{x}$, and thus $Z \in t^{\leftarrow}\left(M^{x}\right) \subseteq M^{\tau(x)}$. So $\tau(x) \in c l_{\beta Y} Z$, 
and hence $x \in \tau^{\leftarrow}\left(c l_{\beta Y} Z\right)$, i.e. $c l_{\beta X} t(Z) \subseteq \tau^{\leftarrow}\left(c l_{\beta Y} Z\right)$. Now we show that $\tau$ is continuous.

First we show that $t\left(0^{\tau(x)}\right) \subseteq 0^{x}$ for every $x \in X$. Let $x \in X$. Consider $A=$ $\left\{P \subseteq Z(X) \mid 0^{x} \subseteq P\right.$, and $P$ is a prime $z$-filter $\}$. Then $t^{\leftarrow}(P)$ is prime for every $P \in A$, and $t^{\leftarrow}(P) \subseteq t^{\leftarrow}\left(M^{x}\right) \subseteq M^{\tau(x)}$ (as $0^{x}$ is contained in only one $z$-ultrafilter, namely $\left.M^{x}\right)$. Thus, by $[1,7.15], t^{\leftarrow}(P) \supseteq 0^{\tau(x)}$ for every $P \in A$. But by $[1,2.8]$ $0^{x}=\bigcap_{P \in A} P$. Therefore $t\left(0^{\tau(x)}\right) \subseteq \bigcap_{P \in A} P=0^{x}$.

Let $x \in \beta X$, and let $W \in 0^{\tau(x)}$ (i.e. $c l_{\beta Y} W$ is a neighborhood of $\tau(x)$ ). Then $t(W) \in 0^{x}$. However, $c l_{\beta X} t(W) \subseteq \tau^{\leftarrow}\left(c l_{\beta Y} W\right)$, and the former set is a neighborhood of $x$ in $\beta X$ (as $\left.t(W) \in 0^{x}\right)$. Thus $\tau^{\leftarrow}\left(c l_{\beta Y} W\right)$ is a neighborhood of $x$. Since the closures in $\beta Y$ of the sets in $0^{\tau(x)}$ form a neighborhood base $\tau(x)$, this shows that $\tau$ is a continuous map.

Suppose $Z \in Z(Y)$ and $t^{\leftarrow}\left(M^{p}\right)$ is maximal for every $p \in \tau^{\leftarrow}\left(c l_{\beta Y} Z\right)$. If $p \in$ $\tau^{\leftarrow}\left(c l_{\beta Y} Z\right)$, then $\tau(p) \in c l_{\beta Y} Z$ and hence $Z \in M^{\tau(p)}$. Since $t^{\leftarrow}\left(M^{p}\right)$ is maximal and is contained in $M^{\tau(p)}$ we must have $t^{\leftarrow}\left(M^{p}\right)=M^{\tau(p)}$. Thus $Z \in t^{\leftarrow}\left(M^{p}\right)$ and so $t(Z) \in M^{p}$ and $p \in c l_{\beta X} t(Z)$. Hence $c l_{\beta X} t(Z)=\tau^{\leftarrow}\left(c l_{\beta X} Z\right)$.

It is evident that distinct homomorphisms from $Z(Y)$ to $Z(X)$ may induce the same map from $\beta X$ to $\beta Y$. All that is required for two homomorphisms $t_{\alpha}$ and $t_{\beta}$ from $Z(Y)$ to $Z(X)$ to induce the same map from $\beta X$ to $\beta Y$ is that given $x \in \beta X$, there is a point $p \in \beta Y$ such that $t_{\alpha}^{\leftarrow}\left(M^{x}\right), t_{\beta}^{\leftarrow}\left(M^{x}\right) \subseteq M^{p}$.

The continuous map $\tau$ induced by $t$ in 3.2 in turn induces a homomorphism $t^{\prime}: Z(\beta Y) \rightarrow Z(\beta X)$ defined by $t^{\prime}(Z)=\tau^{\leftarrow}(Z)$. Thus if $Z \in Z(\beta Y)$, then $t^{\prime}(Z) \cap X=\tau^{\leftarrow}(Z) \cap X \supseteq \tau^{\leftarrow}\left(c_{\beta Y}(Z \cap Y)\right) \cap X \supseteq t(Z \cap Y)$. So if $Z \in Z(Y)$ and $W \in Z(\beta Y)$ such that $Z=W \cap Y$ then $t^{\prime}(W) \cap X \supseteq t(W \cap Y)=t(Z)$.

Thus it can be seen that if $f: X \rightarrow Y$ is continuous, then $t: Z(Y) \rightarrow Z(X)$ defined by $t(Z)=f^{\leftarrow}(Z)$ is a $(\sigma-)$ homomorphism. Then by Proposition 3.2 there is a map $\tau: \beta X \rightarrow \beta Y$ such that $\tau^{-}\left(c l_{\beta Y} Z\right) \supseteq c l_{\beta X} t(Z)=c l_{\beta X}\left(f^{\leftarrow}(Z)\right)$ for any $Z \in Z(Y)$. Thus $\tau$ must agree with $f$ on $X$. For if $\tau(x) \neq f(x)$, let $Z \in Z(Y)$ be such that $f(x) \in Z$ and $\tau(x) \notin c l_{\beta Y} Z$. Clearly $x \in c l_{\beta X}\left(f^{\leftarrow}(Z)\right)$, hence, $x \in$ $\tau^{\leftarrow}\left(c l_{\beta Y} Z\right)$. But then $\tau(x) \in c l_{\beta Y} Z$ contrary to assumption. Therefore $\tau$ is precisely the Stone extension of $f$. Since $t$ is also a $\sigma$-homomorphism it follows that $\tau(v X) \subseteq v Y$.

\section{REFERENCES}

1. L. Gillman and M. Jerison, Rings of continuous functions, Van Nostrand, Princeton, 1960.

2. Mark Mandelker, $F^{\prime}$-spaces and z-embedded subspaces, Pacific J. of Math. 28 (1969), 615-621.

3. Stone, M. H., Applications of the theory of Boolean rings to general topology, Trans, Amer. Math. Soc. 41 (1937), 375-481.

\footnotetext{
Mathematics Department UNIVERSITY OF TORONTO TORONTO ONT.
} 